\title{
Judgments of similarity and spatial models'
}

\section{RAY HYMAN AND ARNOLD WELL}

UNIVERSITY OF OREGON

Ss judged the similarity between all pairs of stimulus objects under 3 conditions: when the objects were (a) Munsell $5 \mathrm{R}$ color patches varying in value and chroma; (b) parallelograms varying in size and tilt; and (c) circles-with-radius varying in diameter and angle of radius. For each set of judgments, the pattern of deviations from the Euclidean model was used to diagnose the most appropriate spatial model. The results confirm previous findings that the Euclidean space is appropriate for judgments of color patches, but that the city block space is appropriate for judgments of geometric forms which vary on perceptually distinct dimensions.

In the present study we investigated judgments of similarity between pairs of objects that differed on two dimensions. We were concerned with the question that was raised by Attneave (1950): How does the overall judgment of similarity depend upon differences on the dimensional components?

Since 1950, two alternative answers have been proposed. Both answers assume that judgments of similarity can be represented by a "spatial model." The assumption of a spatial model emphasizes the perceived difference or dissimilarity between two objects rather than the perceived similarity. The perceived dissimilarity (or the complement of the judged similarity) is treated as if it has the properties of a distance metric. The additional assumption is made that the set of psychological distances between all pairs of specified objects can be embedded within an n-dimensional coordinate space. The question of which spatial model is appropriate, then, is the question of what is the form of the space in which the psychological distances can be embedded. And this question, in turn, reduces to one about the nature of the function which relates perceived distance to perceived differences on the component dimensions.

Attneave (1950) proposed the answer, on the basis of his research, that the appropriate spatial model was of a non-Euclidean form-a form which has subsequently become known as the "city block" model. According to this answer, the perceived distance between two objects can be represented as the sum of the perceived differences on the component dimensions.

Torgerson (1952), following the earlier suggestion of Richardson (1938), concluded that the Euclidean space was appropriate, at least for his data. In the Euclidean model the perceived distance between two objects is related to the component differences by means of the Pythagorean theorem.

These conclusions about the appropriate spatial model are based on two different types of evidence or approaches-the approach of multidimensional scaling and the approach of multidimensional psychophysics. In the scaling approach, the investigator assumes he knows the appropriate spatial model. So far this approach has restricted itself to the Euclidean model. Assuming a Euclidean space, the investigator treats the obtained judgments as distances in an n-dimensional space, and he extracts a number of dimensions sufficient to reproduce adequately the original distances. The appropriateness of the model is determined by how well these dimensions reproduce the original distances. The decision as to whether the "goodness of fit" to the model is adequate is "absolute," in that no comparison is made with a specified alternative model. If the amount of variance accounted for exceeds an arbitrary value -a value which is rarely specified in advance and for which there is no consensual standard-then the Euclidean metric is declared appropriate.

In the psychophysical approach the investigator assumes he knows the component dimensions. He then attempts to directly decide which combinatorial rule or spatial model best describes how $S$ uses these known dimensions in making the overall judgment. The psychophysical approach is a comparative one in that the data are used to decide between two or more alternative models for describing S'S judgments.

Unfortunately, the evidence for and against the Euclidean spatial model is completely confounded by the type of stimulus objects and the type of approach employed. The major evidence for the $\mathrm{Eu}-$ clidean model comes from studies in which the stimulus objects were color patches varying in such dimensions as hue, chroma, and value (Torgerson, 1952; Helm, 1964; Indow \& Kanazawa, 1960; Indow \& Uchizono, 1960). In addition, all the studies with color patches relied entirely on the scaling approach. No attempt was made to see if a specified alternative, such as the city block model, might account for the same data equally well or better.

Surprisingly, at the time of this writing, only two studies exist which employed the psychophysical approach to directly compare alternative spatial models. Both Attneave (1950) and Shepard (1964) used geometric forms as stimulus objects. And both concluded that the Euclidean metric was not appropriate for describing perceived distances between these forms. Attneave concluded that the city block model was appropriate for his data. Shepard suggests that for any one $S^{\prime}$ 's entire set of judgments, because of 
fluctuations in states of attention, no spatial model may be appropriate, but that for any particular state the appropriate spatial model is one somewhere between the Euclidean and the city block.

Attneave (1962), Shepard (1964), and Torgerson (1958) each suggest that the essential difference between conditions under which a Euclidean or nonEuclidean model may be appropriate lies in the nature of the stimulus objects being compared. When the dimensions are "obvious" and "compelling" or "perceptually distinct," such as in the geometric forms varying in size and tilt, then the judgments will fit the additive or city block model. When the dimensions are less distinct and the stimulus objects behave as "unitary wholes," then the Euclidean model will provide the better description of the judgments.

Although the distinction between analyzable and non-analyzable stimulus objects is probably related to the form of the spatial model, the argument for this relationship is somewhat circular at the moment because such a classification of the stimulus materials has been made only on the basis of the outcomes of the original studies. Before this distinction is pursued in more depth, it seems necessary to first confirm the findings about spatial models for color patches and geometric objects under conditions in which the type of approach and type of stimulus materials are not confounded. This latter objective is the basis for the present study.
In the present study our goal was to remove the confounding of stimulus materials and method by employing three different types of stimulus objects -color patches, parallelograms, circles-with-radius -which have been used in the previous key studies on spatial models. We would thus have the same $S$ make judgments on all three types of stimulus material, and we could compare spatial models in terms of a uniform set of criteria. In addition, we could add further refinements and improvements to gain a better understanding of how judgments of similarity differ for these different stimulus objects.

Basically, our approach combines features of both the scaling and the psychophysical approaches. We used the techniques of multidimensional scaling to fit the Euclidean model to the obtained similarity judgments. We employed the psychophysical approach by assuming that we knew the two dimensions that $S$ was using in making his judgments. Given this assumption, we could then study the pattern of deviations from the Euclidean baseline to decide if the judgments were Euclidean or if they were closer to an alternative model such as the city block.

We can schematically illustrate some aspects of both the theoretical basis and our approach with the aid of Fig. 1. A standard stimulus object (St) is located at the extreme left on a display board. $S$ is asked to place each of a set of comparison objects (C1) such that the horizontal distance from the left

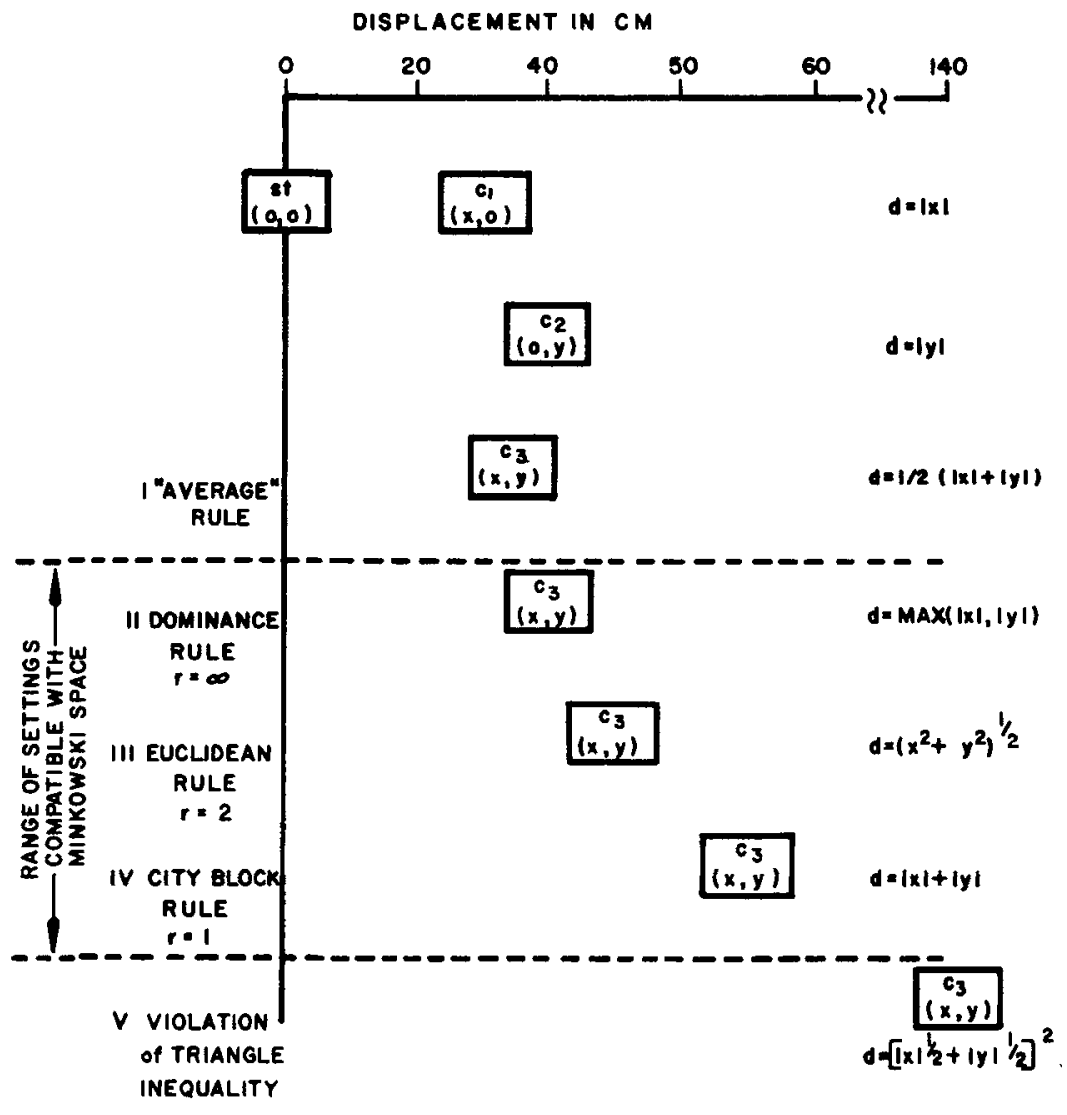

Fig. 1. Schematic representation of procedure for obtaining distance judgments. Several settings of a bidimensional comparison stimulus, each setting corresponding to a possible rule of combination, are illustrated. 
hand side of the board to the comparison object indicates the perceived distance (cf., Indow \& Uchizono, 1960). In the hypothetical example of Fig. 1 , $\mathrm{S}$ has placed $\mathrm{Cl}$, which differs on only the first dimension from St, $30 \mathrm{~cm}$ to the right of the standard; he has placed C2, which differs only on the second dimension, $40 \mathrm{~cm}$ to the right of the standard. These placements indicate that for this $S$ on this trial a difference of $x$ units on the first dimension is psychologically $3 / 4$ of a difference of $y$ units on the second dimension.

Knowing the perceived difference for an amount $x$ on the first dimension and an amount $y$ on the second dimension, the relevant question becomes: What will the setting be for a comparison object that differs from St simultaneously by amounts $x$ and $y$ on the first and second dimensions? If $\mathrm{S}$ combines differences on the two dimensions by a consistent rule, what is the rule? Figure 1 illustrates a range of possible settings for the bidimensional comparison C3, each comparison consistent with a different rule. At one extreme is a rule in which $S$ makes his overall judgment by averaging the perceived differences on each dimension (Setting I). At the other extreme is a rule whereby $S$ exceeds the sum of the component differences to arrive at the overall dissimilarity (Setting $V$ ). Many other rules are, of course, possible, but Settings I to $\mathrm{V}$ include the range of actual settings that were made throughout our experiments. Rarely, if ever, was S observed making a setting that was as extreme as either of these limits.

Only some of the possible rules within this range of settings would be consistent with a spatial model. Setting III is consistent with the Euclidean model and Setting IV is consistent with the city block model. Setting II is also consistent with a spatial model, one which we will call the "dominance" model because the judgment, on each comparison, is made entirely in terms of the one dimension that is perceptually "dominant" for that pair. Settings II and IV represent extremes that would be consistent with the generalized distance metric known as the Minkowski r-metric. In the class of Minkowski r-spaces, the distance between any two points $i$ and $j$ is given by the equation:

$$
d(i, j)=\left[\sum_{m}^{p}\left(\left|a_{i m}-a_{j m}\right|\right)^{r}\right]^{1 / r} \quad r \geq 1
$$

where $a_{i m}-a_{j m}$ is the difference between stimulus object $i$ and stimulus object $j$ on dimension $m$; $p$ is the number of orthogonal component dimensions. When $r$ is 2, the equation becomes the familiar Pythagorean formula for distance in the Euclidean space (Setting III). When $r$ is 1 , the space is the city block one (Setting IV). When $\mathbf{r}$ goes to infinity we have the dominance model (Setting II). Settings II through IV merely represent three points on a con- tinuum of possible spaces between the range of values of $r$ from infinity to 1 . A setting such as I violates the spatial metric because the overall distance is less than one of the perceived component distances; and a setting such as $\mathrm{V}$ violates the triangle inequality in that the overall distance is more than the sum of the component distances.

Figure 1 suggests a direct method for testing S's combinatorial rule. We did not use such a direct criterion in Experiment I because the configuration of the stimuli on the component dimensions did not allow for the separate determination of unidimensional and bidimensional components. We were able to employ such a criterion in Experiment II to supplement our major criterion. Our major criterion was based on a heuristic consideration. We used the Euclidean metric as the baseline or common yardstick against which to study the pattern of judgments for all three types of stimulus material. We reasoned that if $\mathrm{S}$ made his judgments according to the city block model, then, relative to his unidimensional judgments, he would judge bidimensional differences as being larger than would be predicted from the Euclidean model (cf., Settings III and IV). Consequently, when we studied the pattern of deviations from the Euclidean model, we should find, in the case of the city block model, that the average deviation of bidimensional comparisons should be relatively positive and the average deviation of the unidimensional comparisons should be relatively negative. Thus, if the $\mathrm{S}$ were truly city block, the difference between the average deviations of bidimensional and unidimensional comparisons should be positive. Conversely, if $S$ deviated from the Euclidean model towards the dominance model, then the difference -average deviation for bidimensional minus average deviation for unidimensional comparisons-should be negative.

Actually, we expected to observe three types of deviations from the Euclidean metric: (a) nonsystematic deviations which would represent unreliability in judgments; and (b) systematic deviations of two types. One kind of systematic deviation, as we have already mentioned, would be indicated by a differential pattern of deviations of distances between objects differing on only one of the component dimensions and distances between objects differing on both components. A third type of deviation would be systematic deviations due to discrepancies in the obtained settings and S's actual perceived distances. Helm and Tucker (1962) and Indow (1963) have reported systematic deviations from the Euclidean model which they attribute to defects in the method of measuring the psychological distances rather than the spatial model. For example, if $\mathrm{S}$ systematically underestimates the large distances relative to the small distances (because, say, the width of the board forces him to foreshorten the large distances), this will 


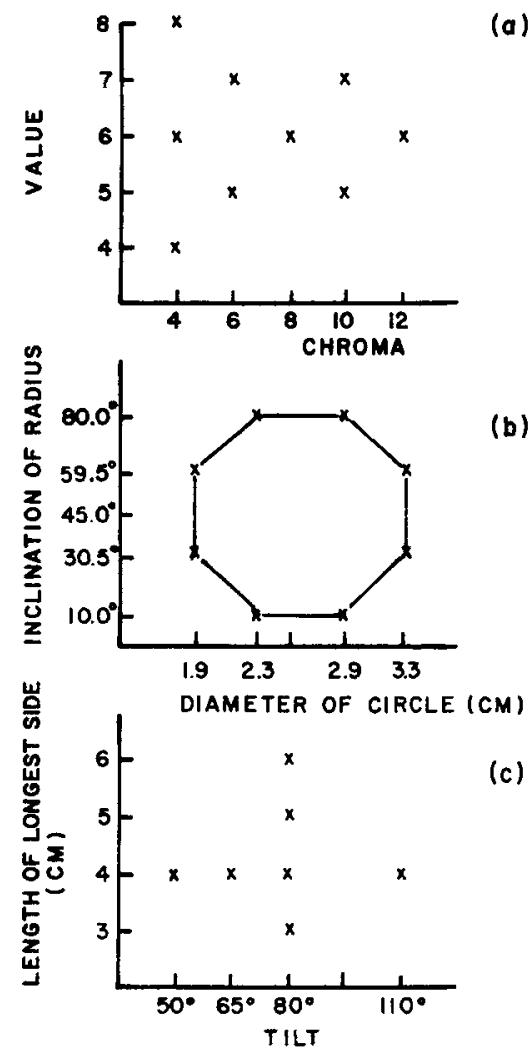

Fig. 2. Configurations of the three stimulus sets upon their component dimensions: (a) the coordinates of the nine Munsell $5 R$ patches used by Torgerson (1952); (b) the coordinates of the eight circles-with-radius used by Shepard (1964); and (c) the coordinates of the seven parallelograms used by Attneave (1950).

produce a characteristic pattern of deviations. Our hope was that, to the extent such defects of the distance model affected the judgments, they would do so Independently of the deviations due to the spatial model. Our results seem to be consistent with this expectation.

\section{EXPERIMENT I}

Experiment I was conducted to see if the conclusions about spatial models drawn by Torgerson (1952), Attneave (1950), and Shepard (1964) can be confirmed with stimulus objects of the same type, number, and configurations that were employed respectively in these separate investigations. In particular, when the judgments of each $S$ are analyzed separately and according to a common criterion, will judgments of the color patches conform to the Euclidean model, and will judgments of the geometric stimuli conform to the city block or some model between the city block and the Euclidean?

\section{Subjects}

Six Ss were hired through the University of Oregon student employment service and were paid at the rate of $\$ 1.50$ per hour. The Ss were required to have normal color vision as determined by the Ishihara test for color blindness. Each $\mathrm{S}$ attended four sessions of approximately $1 \mathrm{hr}$. and $45 \mathrm{~min}$. in duration. One of the authors, $\mathrm{RH}$, was also run through the four sessions and he is included as a seventh $S$.

\section{Stimulus Objects}

Four sets of stimulus objects were used. Sets I and II consisted of patches of the nine Munsell 5R colors which were used by Torgerson (1952) (see Fig. 2a). These patches, obtained from the Munsell Color Company, had a matte finish, and were $1.2 \times 1.7 \mathrm{~cm}$. Stimuli in Set I were mounted on $2 \times 3$ in. black cards and those in Set II were mounted on $2 \times 3$ in. white cards.

Set III consisted of a set of circles-with-radius Identical in number and coordinate values with those used by Shepard (1964). (See Fig. 2b). These consisted of eight circles each with a radius drawn in, varying both in area and inclination of the radius. The figures were drawn in black ink on $3 \times 5 \mathrm{in}$. white file cards.

Set IV consisted of seven parallelograms varying in size and tilt (Fig. 2c). These were drawn in black ink upon white file cards so as to be identical in size and shape to those employed by Attneave (1950); Attneave's actual stimulus objects differed from ours in that his were cut out of colored paper-six of them were blue and one was purple.

\section{Precedure}

Our procedure was based on Indow's Method of Multiple Ratios (Indow \& Uchizono, 1960). The stimulus objects were presented, one set per session, on a board similar to that described by Indow and Uchizono. The board was constructed of stiff white cardboard, $30 \times 40$ in. Fixed to the board were eight horizontal wooden trays onto which the stimulus objects were placed. The board rested upon a table such that the surface facing $S$ was tilted away from him approximately 10 degrees from the vertical plane. For each trial, one stimulus was designated as the "standard" and was placed in one of the middle trays $^{2}$ at the extreme left of the board. The other n-1 stimull were placed, one per tray, in a vertical column at the right side of the board. The S was instructed to move these n-1 comparison stimuli such that the more similar to the standard a stimulus was judged to be, the further to the left end of the board it was to be placed. The actual instructions were as follows:

You see in front of you a board with a number of cards in the trays. This card will be the standard for this trial (indicate). Your task is to move the other cards so as to satisfy the rule that the horizontal distance between the standard and any other card indicates the degree of simi- 
larity between them. That is, if two cards are very similar in appearance, there will be very little horizontal distance between them. You are to get up and move the cards so as to satisfy this rule and then return to your chair. You may repeat this procedure as often as you like until you are satisfied; however, you will have to stop after a maximum of five minutes has passed. Are there any questions?

During an experimental session, each stimulus object assumed the position of the standard. A session consisted of $n$ plus two trials with one of the four sets of stimulus objects. The first and fifth trials were repeated as checks on S's consistency. Only the second of the repeated trials was used in the data analysis. The order in which the different stimulus objects became the standard as well as the vertical positions of the comparison stimuli were determined by reference to tables of random numbers. A different randomization was used for each $S$ and for each session.

The $S$ began each trial seated on a chair placed $6 \mathrm{ft}$. from the board. He made his judgments by leaving his chair and moving the comparison stimuli the appropriate horizontal distances. After making these judgments the $S$ returned to his chair. He was free to make as many readjustments as he felt were necessary but was required to complete each trial within $5 \mathrm{~min}$.

After each trial, the horizontal distance between the center of each comparison card and the center of the standard card was measured and recorded to the nearest tenth of a centimeter. The next standard was then placed in position, the other cards randomized, and the next trial commenced.

Each $S$ was run individually in an experimental room in which the windows were covered to secure relatively uniform lighting. The room was lighted by four overhead 40 W General Electric fluorescent lamps and was painted a dull white. The amount of illumination impinging upon the surface of the test board was estimated to be approximately $35 \mathrm{ft} .-\mathrm{c}$.

Each S attended four sessions. The first and fourth sessions for all Ss were devoted to the color chips. Half of the Ss had Set I (black background) and half had Set II (white background) in the first session. Sessions 2 and 3 for all Ss were devoted to the geometric stimuli. Some Ss received the Attneave stimuli (Set IV) in Session 2 and the Shepard circles (Set III) in Session 3, and some received them in the reverse order.

\section{Results}

Each S's settings for a session were converted into symmetric distances by the procedure of Indow and Uchizono (1960). The matrix of symmetric distances was then fitted to a Euclidean set of coordinates according to the procedures suggested in
Torgerson (1958). The vectors corresponding to the first two principal components were used as the basis for judging how well, and in what manner, the original judgments could be represented in a Euclidean plane. This part of the analysis, as well as all subsequent analyses, were programmed for, and run on, an IBM $360 / 50$ computer.

Reliability of Judgments. The internal consistency of S's judgments was measured by the correlation between the judgments of the same pairs of stimuli $i$ and $j$, one member of the pair being the judgment when $i$ was the standard and other member being the judgment when $j$ was the standard. These correlations for each stimulus condition are reported in Table 1. The reliability of the average of these two judgments as estimated by the Spearman-Brown formula is reported in the second column of Table 1 .

Because these correlations are both a function of how consistent $S$ is from judgment to judgment as well as of how much relative spread there is between the perceived distances of close and far-apart stimuli, Table 1 also reports coefficients of variation in order to facilitate comparisons among the stimulus sets in terms of relative consistency of judgment and relative variability in distances.

Although the geometric stimuli tend to yield higher reliabilities and show more internal consistency, these differences were not statistically significant. Despite the differences in configurations, the relative spread between stimulus pairs seems to be about the same for all stimulus types with the possibility that the variation between small and large distances within the Shepard stimuli is somewhat smaller.

Goodness of Fit. As one criterion of goodness of fit to the Euclidean model, Torgerson (1958) suggests the proportion of variance among the scalar products accounted for by the first $\mathrm{p}$ Euclidian vectors. Table 2 provides this index, based on the first two Euclidean vectors, for the stimulus materials of Experiment I.

Table 1. Indices of reliability and consistency within and between judgments of stimulus pairs.

The entries are medians based on seven Ss. Experiment 1 .

\begin{tabular}{lcccc}
$\begin{array}{c}\text { Stimulus } \\
\text { Set }\end{array}$ & $\begin{array}{c}\text { Correlation } \\
\text { between }(i, j) \\
\text { and }(i, i)\end{array}$ & $\begin{array}{c}\text { Reliability } \\
\text { symmetric } \\
\text { distance }\end{array}$ & $\begin{array}{c}\text { Relative }^{b} \\
\text { error in } \\
\text { judgment }\end{array}$ & $\begin{array}{c}\text { Relative }^{c} \\
\text { variability } \\
\text { in distances }\end{array}$ \\
\hline Colors 1d & .78 & .88 & $19 \%$ & $52 \%$ \\
Colors 2 & .80 & .89 & $16 \%$ & $51 \%$ \\
Attneave & .89 & .94 & $13 \%$ & $50 \%$ \\
Shepard & .90 & .95 & $9 \%$ & $40 \%$ \\
\hline
\end{tabular}

a Based on Spearman-Brown formula applied to correlations between corresponding elements in asymmetric matrix.

$b$ Coefficient of variation computed by taking the estimate of the standard deviation between distances which is due to unreliability as a ratio of the average distance.

$c$ Coefficient of variation computed by taking the estimate of the true standard deviation between perceived distances as a ratio of the average distance.

$d$ Here 1 and 2 refer to the order in which $S$ received the stimulus set. 
Table 2. Proportion of the variance of scalar products accounted for by the first two Euclidean vectors. Experiment $I$.

\begin{tabular}{lcccc} 
& \multicolumn{4}{c}{ Stimulus Set } \\
& Colors 1 & Colors 2 & Attneove & Shepard \\
\hline Median: & $94.3 \%$ & $95.1 \%$ & $95.8 \%$ & $93.2 \%$ \\
Worst: & 86.7 & 90.9 & 85.0 & 84.4 \\
Best: & 96.0 & 96.2 & 98.0 & 98.5 \\
\hline
\end{tabular}

At the moment there is no accepted standard for deciding when such a percentage is sufficiently high to warrant the appellation "good fit." Reports from various studies in the literature, each one concluding that its data fit the Euclidean model, range from 94.4 to $98.1 \%$ (Helm, 1964; Indow \& Kanazawa, 1960; Indow \& Uchizono, 1960; Torgerson, 1958). Considering the fact that our data are based on fewer judgments per stimulus pair and are not pooled over Ss, we can conclude that, on the basis of this index, our data fit the Euclidean model as well as data from other studies in the literature.

But the most important implication of the results in Table 2 is that by this criterion, the geometric stimuli-ones which have yielded non-Euclidean spaces by other methods in previous studies-fit the Euclidean model at least as well as the color patches-stimuli which have consistently yielded "good fits" to the Euclidean modsl in previous studies.

Systematic Deviations from the Euclidean Model. As we have mentioned, we expected to find two types of systematic deviations from the Euclidean distances as recovered from the first two principal components. One type of deviation would be attributable to defects in the method of measuring psychological distance. For example, in about $20 \%$ of the color sessions, Ss showed a pattern of deviations in which mediumsized distances were systematically underestimated relative to the small and large distances. Such a pattern could come about if $S$ underestimated the middle distances and emphasized the extremes at both ends. Among some Ss we observed a strategy which tended to result in just this sort of behaviorthe $\mathrm{S}$ would first dichotomize all the comparisons into those that are "like" the standard and those that are not. Then he would separately adjust stimuli in each subgroup in terms of their apparent distance from the standard. Another systematic pattern occurring with equal frequency within the color sessions was a tendency for $S$ to underestimate the smaller distances relative to the larger distances. In the majority of cases, however, we detected no such systematic patterns. And in the cases where these types of deviations due to the judgment procedure existed, they did not seem to obscure or bias the discovery of the type of systematic deviation that we were looking for with respect to spatlal models.

Table 3 reports the results based on the type of systematic deviations that we predicted would reflect effects due to non-Euclidean combinatorial rules. We expected that city block judgments would tend to result in positive deviations from the Euclidean model for comparisons involving two dimensions-because the combining rule would yield a distance larger than that predicted by the Euclidean model (see Fig. 1). To compensate for this, since the model is fitted to all the data, we expected a counterbalancing tendency for all the unidimensional comparisons to yield distances that deviate negatively from the Euclidean model. Thus, we expected that the difference between the average deviation of bidimensional distances and the average deviation of unidimensional distances would be significantly positive when S's judgments conformed to the city block model.

Table 3 reports the means based on this criterion as well as the results of a combined test of significance. The null hypothesis being tested is that all these means are zero. The larger the means are in the positive direction, the more the pattern of judgments deviates from a Euclidean model towards the city block model. As predicted from previous research, the criterion suggests that the colors are consistent with the Euclidean model and that the Attneave and the Shepard stimuli are inconsistent with the Euclidean model and probably fit some model closer to the city block. Not one of the 14 critical ratios for individual $\mathrm{Ss}$ in the two color sets is significant, although one $S$ achieved critical ratios as high as 1.93 and 1.66 . Nor did any of the seven critical ratios for judgments of the Attneave stimuli reach significance, the largest being 1.46 . However, six of the seven were positive and the negative one was -0.43 . On the other hand, five of the seven critical ratios for the Shepard stimuli were positive and significant beyond the .01 level (ranging grom 2.94 to 4.04); of the two non-significant ones, one was negative $(-1.32)$.

Pattern of Deviations from the Euclidean Model: Qualitative. The results in Table 3 must be qualified since the stimulus sets differ in the number of ob-

Table 3. Deviations from Euclidean distance: Average deviation of bidimensional minus average deviation of unidimensional judgments. Experiment 1.

\begin{tabular}{lcccc} 
& \multicolumn{3}{c}{ Stimulus Set } \\
& Colors 1 & Colors 2 & Attneave Shepard \\
\hline $\begin{array}{l}\text { Mean difference } \\
\text { Pooled critical } \\
\text { ratio (Mann-Whitney) }\end{array}$ & $2.4 \mathrm{~cm}$ & $1.7 \mathrm{~cm}$ & $3.3 \mathrm{~cm}$ & $11.5 \mathrm{~cm}$ \\
\hline
\end{tabular}

* Significant at the .05 level.

* Significant at the .01 level.

a A Mann-Whitney test based on rank order of deviations of uniand bidimensional judgments was performed separately for each $S$ and stimulus set. The pooled critical ratio is obtained by combining the separate critical ratios for the seven $S$ s according to the procedure suggested by Mosteller and Bush (1954). 
jects and in their configurations. The average deviation criterion was based on the assumption that for Ss whose judgments were in perfect agreement with the city block model there would be no overlap in the deviations of bidimensional and unidimensional deviations from the Euclidean baseline. As our simulations revealed, however, this was true only for the configuration employed with the Shepard stimuli. The overlap for a simulated city block $S$ with the Attneave stimuli, for example, is such that the MannWhitney $z$ on differences between bidimensional and unidimensional deviations was only 1.60 . Thus, even if S's judgments were perfectly city block, the present criterion when used with Attneave's conflguration would be powerless to detect this on a single S.

Figure 3 shows a comparison of the results from two of our Ss with corresponding simulations of hypothetical Ss who obey either the dominance model, Fig. 3a, or the city block model, Fig. 3c. The horizontal line indicates the size of the Euclidean distances (E') as reconstituted from the first two Euclidean vectors by means of the Pythagorean theorem. On the ordinate are plotted the algebraic deviations of the actual distances (A) minus the re- covered distances (E'). In general the deviations from the model will be larger for the smaller sizes of $E^{\prime}$ since the model is fitted by a least-squares procedure which gives most weight to the larger distances. A point above the line indicates that $S$ overestimated this distance with respect to the $\mathrm{Eu}-$ clidean model. To illustrate, the data from two of our Ss are compared with these simulations. As an aid to visualizing the extent of similarity in the patterns, lines have been separately fitted by means of the method of averages, to the deviations of the unidimensional and the bidimensional judgments. In the case of SVB, for example, the fitted lines suggest his judgments were close to the Euclidean model. The only systematic deviations probably come about because $S$ bunched the comparison objects near the standard together. PR's data also supported the Euclidean model. Again we see systematic deviations due to the measuring procedure rather than departure from the spatial model. In this latter case, the middle distances were underestimated with respect to both extremes. One of the E's qualitatively classified the 14 separate graphs of the sort illustrated in Figs. $3 \mathrm{~g}$ and $3 \mathrm{~h}$ (two for each of the seven (a)

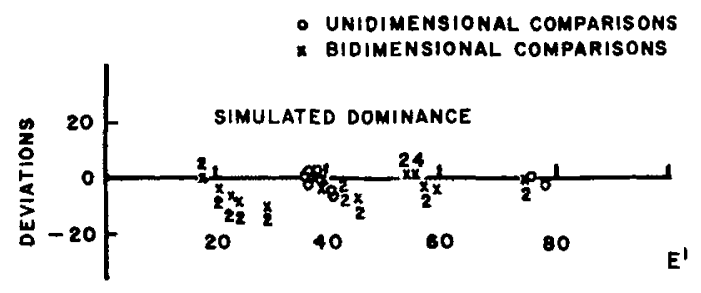

(b)

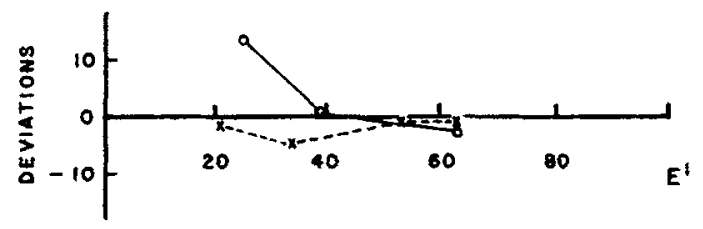

(c)

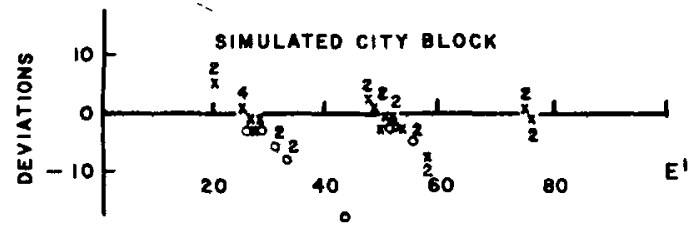

(d)

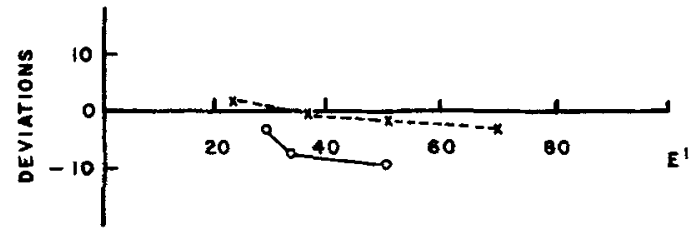

(e)

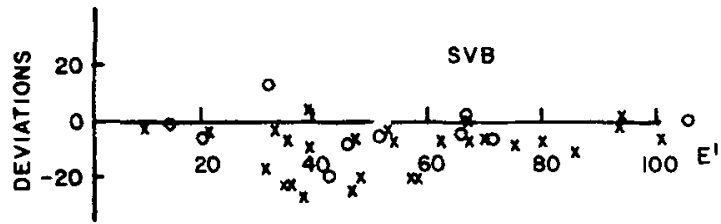

(f)

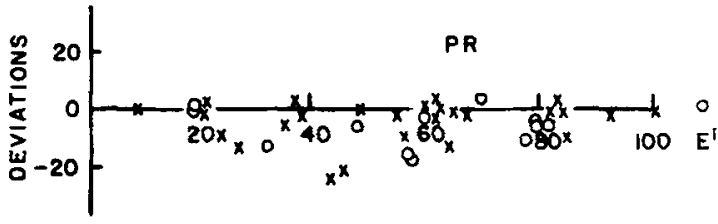

(g)

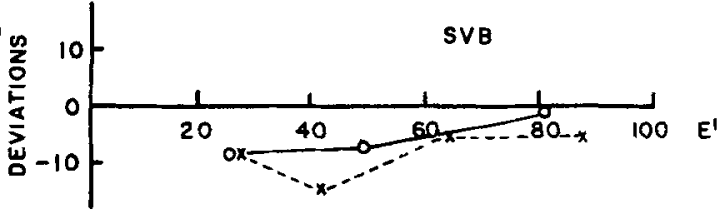

(h)

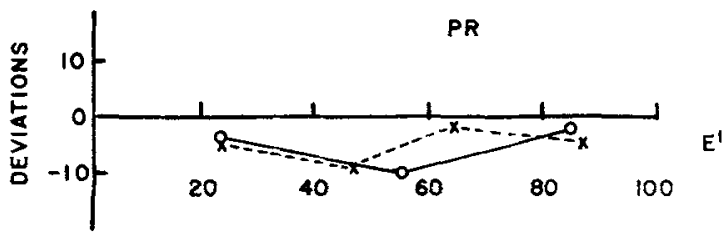

Fig. 3. Pattem of deviations from the Euclidean model for simulated and actual Ss on the color patches: (a) deviations of judgments from the recovered distances for a simulation of the dominance model; (b) the trend of these deviations for unidimensional and bidimensional judgments fitted by the method of averages; (c) deviations of judgments from the recovered distances for a simulation of the city block model; (d) the trend of these deviations for the unidimensional and bidimensional judgments fitted by the method of averages; (e) and (f) the actual deviations of the judgments from two representative $S s ;(g)$ and $(h)$ the trend of the unidimensional and bidimensional deviations of these two S5 fitted by the method of averages. 


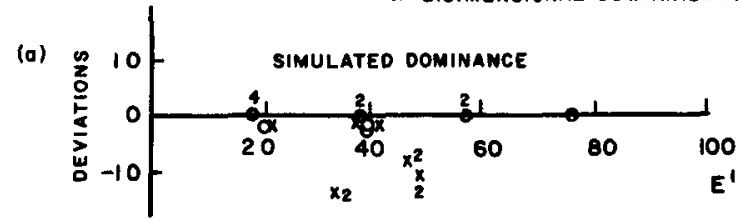

(b)

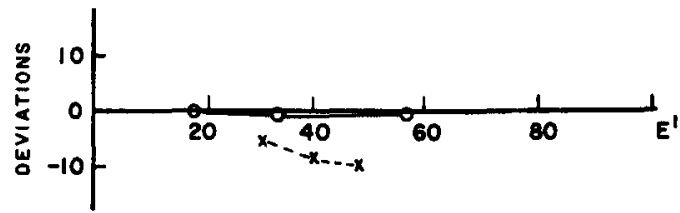

(c)

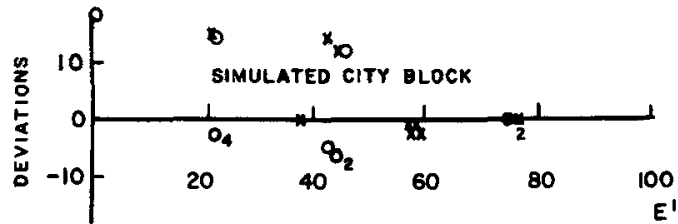

(d)

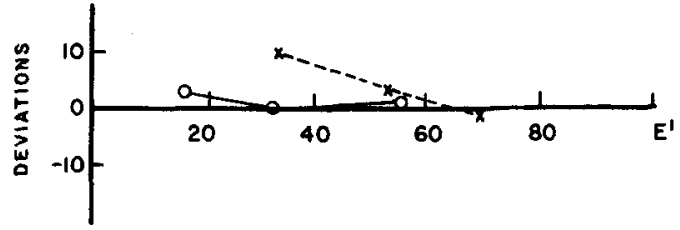

(e)

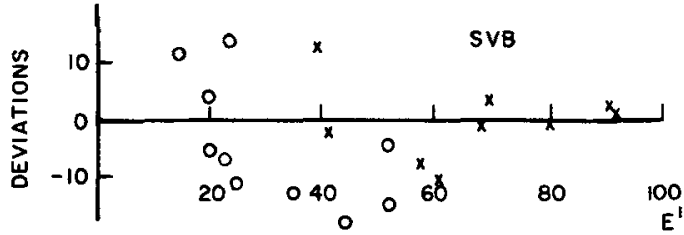

(f)

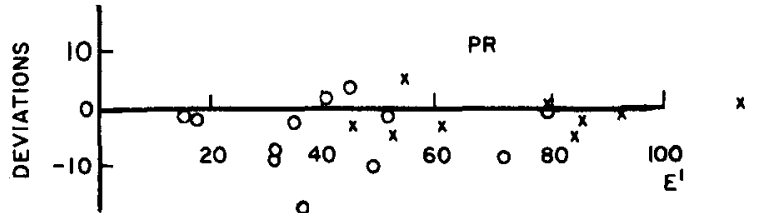

(g)

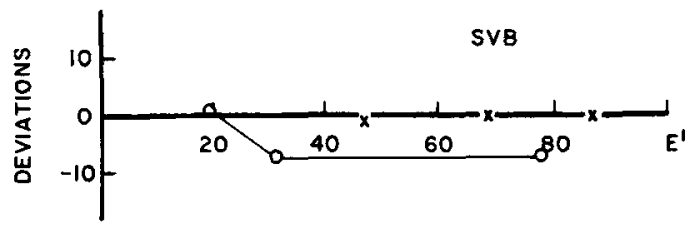

(h)

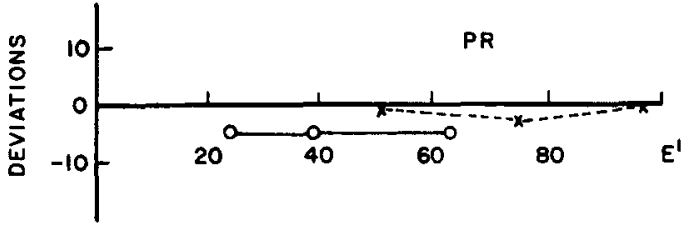

Fig. 4. Pattem of deviations from the Euclidean model for simulated and actual Ss on the Attneave parallelograms: (a) deviations of judgments from the recovered distances for a simulation of the dominance model; (b) the trend of these deviations for the unidimensional and bidimensional judgments fitted by the method of averages; (c) deviations of judgments from the recovered distances for a simulation of the city block model; (d) the trend of these deviations for the unidimensional and bidimensional judgments fitted by the method of averages; ( $e$ ) and ( $f$ ) the actual deviation of two Ss; $(\mathrm{g})$ and $(\mathrm{h})$ the trend of the unidimensional and bidimensional deviations for these two Ss fitted by the method of averages.

Ss) in terms of whether the pattern looked closest to the dominance, Euclidean, or city block simulations. These classifications were made without knowledge of the results on other criteria. By this criterion, 11 of the 14 graphs were classified as closest to the Euclidean model, one was closest to the pattern for dominance, and two were closest to the pattern for city block.

Figure 4 presents the graphic picture for the Attneave data. A look at Fig. 4d tells us why the difference between the average deviations for the bidimensional and unidimensional judgments might underestimate $S^{\prime} s$ actual compatibility with the city block metric. Attneave's configuration is such that at small distances the unidimensional stimuli tend to be overestimated. As a result, even for the perfect city block case, the critical ratio for the difference in rank orders of bidimensional and unidimensional stimull is oniy 1.46. The deviations for SVB have a pattern which seems to fit reasonably close to the simulated city block model; the critical ratio for these differences was 1.42. PR's pattern (Fig. 4h), on the other hand, does not seem to fit the city block pattern. This is supported by a critical ratio of only 0.18. Of the seven patterns on the Attneave stimuli, four were judged to be closest to the city block and the remaining three were judged to be consistent with the Euclidean metric.

Figure 5 illustrates the kind of patterns we obtained with the Shepard stimuli. Both the simulations for the dominance and the city block models produced complete separation of the unidimensional and bidimensional deviations. This suggests that a configuration which is symmetric around both axes may be optimal for deciding between spatial models. The patterns produced by SVB and PR are quite typical of all but one $S$; this one exception produced a pattern that was judged to be consistent with the Euclidean metric.

\section{Conclusions to Experiment I}

Using the goodness-of-fit criterion of Torgerson (1958)-the proportion of variance of scalar products accounted for by the flirst two Euclidean vectorswe found that all three types of stimulus objects produced judgments that fit the Euclidean metric equally well. Indeed, for only three out of seven Ss was one of the color sets best in its fit to the Euclidean metric. 
But this criterion is at best a crude yardstick. It is affected by such extraneous factors as the configuration of the stimuli, the number of stimuli, the relative dispersion among the stimuli, the tendency of $S$ to welght one dimension more than another, inconsistency in judgments, distortions such as end effects in Ss production of indices of his similarity, as well as actual deviations from the Euclidean spatial model.

When we shift from this gross measure to the pattern of deviations from the Euclidean model, we find that, in general, the results support conclusions based on earlier work. The similarity judgments of color patches were consistent with a Euclidean combining rule. And the pattern of deviations of bidimensional and unidimensional judgments for the Attneave and Shepard stimuli differed significantly from the Euclidean baseline towards the city block model.

This result was much more convincing for the Shepard stimuli than for the Attneave. The configuration of the Attneave stimuli, however, is such as to bias the results against a strong deviation from the Euclidean model.

The results also seem to eliminate some possible explanations for the differences among these stimulus sets.
For example, neither indices of reliability of judgment or relative spread among the stimulus objects within a set seem to account for the differences. And, of course, the differences can no longer be attributed to artifacts introduced by pooling over $\mathrm{Ss}$, method of collecting the judgments, or criteria.

The ambiguous results with the Attneave stimuli leave us uncertain as to how much of the observed differences might be attributed to differences in the configurations of the stimulus objects on their component dimensions. Consequently, we felt it necessary to explore this possibility in our next experiment.

\section{EXPERIMENT II}

Experiment II was designed as a replication of Experiment I, but with the three stimulus sets equated both for size and the configuration on the component dimensions. The configuration of the Shepard stimuli in Experiment I was chosen because it provides complete separation between deviations of bidimensional and unfdimensional judgments when either the dominance or the city block model is appropriate. One attractive feature of the octagonal arrangement is that on each trial there is always (a)

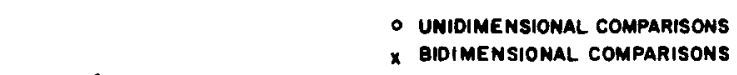

(b)

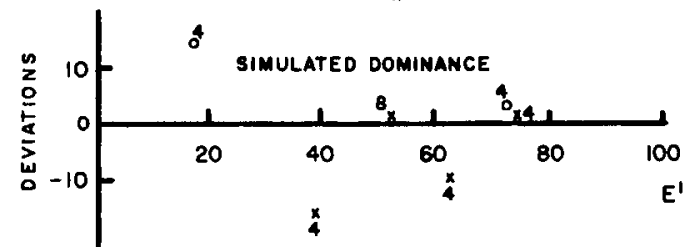

(c)

(d)

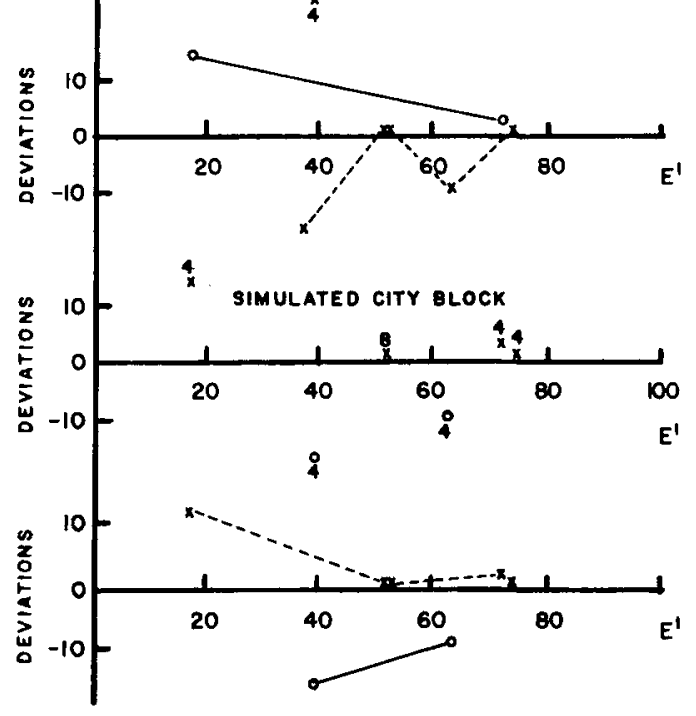

(e)

(f)

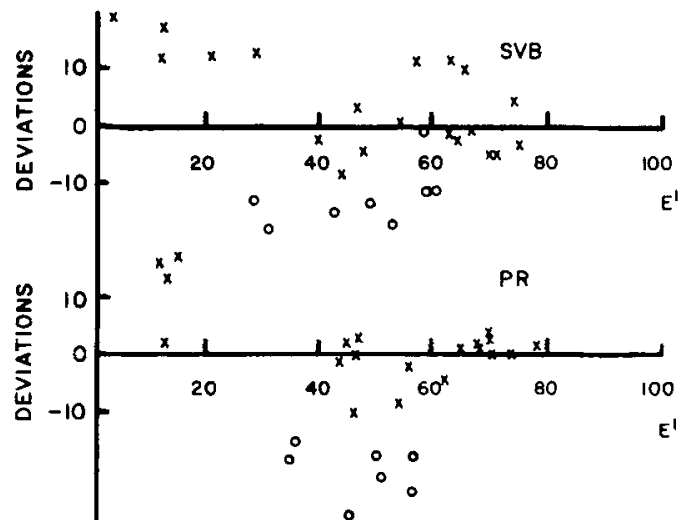

(g)

(h)

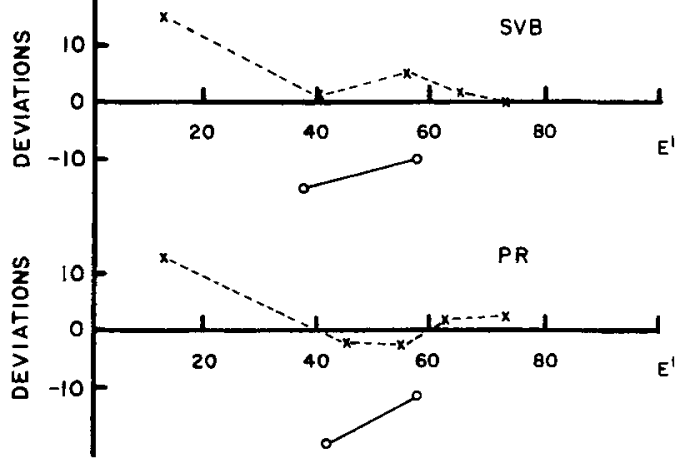

Fig. 5. Pattern of deviations from the Euclidean model for simulated and actual Ss on the Shepard circles-withradius: (a) deviations of judgments from the recovered distances for a simulation of the dominance model; (b) the trend of these deviations for the unidimensional and bidimensional judgments fitted by the method of averages; (c) deviations of judgments from the recovered distances for a simulation of the city block model; (d) the trend of these deviations for the unidimensional and bidimensional judgments fitted by the method of averages; (e) and (f) the actual deviations of two Ss: $(g)$ and $(h)$ the trend of the unidimensional and bidimensional deviations for these two Ss fitted by the method of averages. 
a triad of comparison stimuli which meet the conditions depicted in Fig. 1; i.e., there is a comparison that differs from the standard only on the first dimension by $x$ units; another comparison that differs only on the second dimension by $y$ units; and there is a third comparison that differs simultaneously on both dimensions by amounts $x$ and $y$, respectively. This enables us to determine, for each trial, how close $S$ is approximating one of the settings illustrated in Fig. 1. For each $S$, then, we obtain eight independent estimates of the combinatorial rule; these estimates supplement our findings based on the pattern of deviations.

\section{Subjoets}

\section{METHOO}

Six new Ss were obtained and paid at the same rate as in Experiment I. Whereas all the Ss in Experiment I were undergraduates, five $\mathrm{Ss}$ in this experiment were graduate students, four of them in psychology.

\section{Stimulus Objects}

Three sets of stimulus objects were used. Set I consisted of eight cards on which were mounted $2 \times 2 \mathrm{~cm}$. squares of glossy 5R colors (obtained from the Munsell Color Company). The values on the two Munsell dimen-
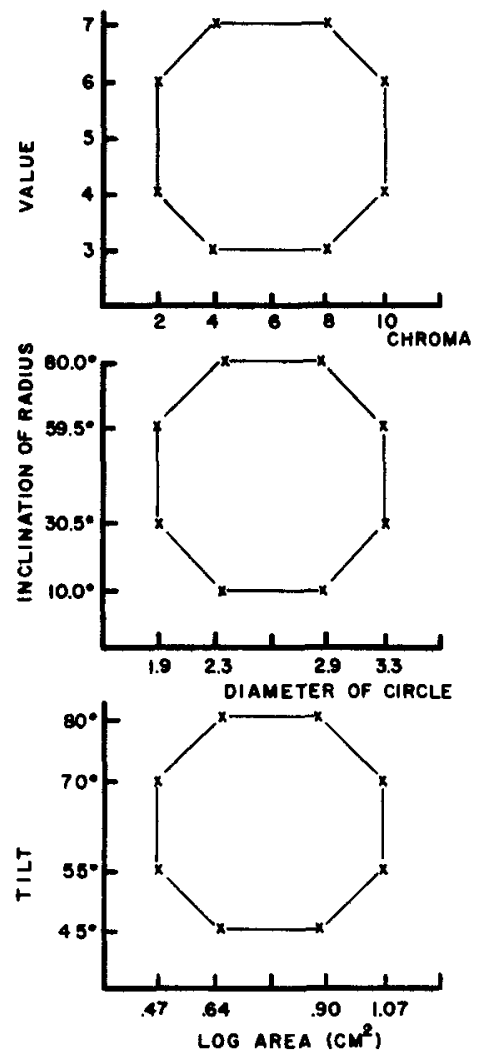

(a)

(c)

Fig. 6. Configurations of the color patches and Attneave parallelograms upon their component dimensions in Experiment II: (a) the coordinates of the eight Munsell 5R color patches; (b) the coordinates of the eight Shepard circles; (c) the coordinates of the eight parallelograms.
Table 4. Indices of reliability and consistency within and between judgments of stimulus pairs.

The entries are medians based upon six Ss. Experiment II.

\begin{tabular}{lcccc}
$\begin{array}{l}\text { Stimulus } \\
\text { Set }\end{array}$ & $\begin{array}{c}\text { Correlation } \\
\text { between }(i, i) \text { of symmetric } \\
\text { and }(i, i)\end{array}$ & $\begin{array}{c}\text { Reliability } a \\
\text { distance }\end{array}$ & $\begin{array}{c}\text { Relative } b \\
\text { error in } \\
\text { iudgment }\end{array}$ & $\begin{array}{c}\text { Relative } \\
\text { spread in } \\
\text { distance }\end{array}$ \\
\hline I. Colors & .80 & .89 & $15 \%$ & $42 \%$ \\
II. Attneave & .92 & .96 & $8 \%$ & $42 \%$ \\
III. Shepard & .78 & .88 & $12 \%$ & $36 \%$ \\
\hline
\end{tabular}

a Based on Spearman-Brown prophecy formula applied to correlations between corresponding elements in asymmetric distance matrix.

$b$ too times ratio of standard deviation of error in distance judgments over average distance between pairs.

c 100 times ratio of standard deviation between "true" distances over average distance between pairs.

sions were selected to be as close as possible to a configuration that would approximate an equilaterial octagon. The limited number of steps available in the Munsell system made the achievement of an actual equilateral octagon impossible. Set III consisted of eight parallelograms similar to those used in Experiment I, but with coordinates chosen to form an equilateral octagon. Since Attneave (1950) discovered that Ss responded to the parallelograms in terms of logarithm of area rather than length of side, we used logarithm of area as the size dimension. Set II consisted of the same circleswith-radius as Set III in ExperimentI. The arrangement of the stimulus sets upon their component coordinates is depicted in Fig. 6.

\section{Procedure}

The data were collected and analyzed exactly as in Experiment I. In addition to the instructions of Experiment I, E explicitly mentioned that the stimulus objects differed from each other in two ways; $S$ was then urged to use both types of differences in making his overall judgment of similarity. This added proviso was inserted to guard against the possibility that $S$ would fall back upon using just one dimension. In the latter case, no information is provided about spatial models. The relevant question of the experiment is a conditional one: when $\mathrm{S}$ employs both dimensions in his judgment, by what rule of combination does he put them together?

Each $S$ attended three separate sessions of approximately one and three-quarters hours in length. Each session was devoted to one of the three stimulus sets. The order of these sets was separately randomized for each $\mathrm{S}$.

\section{Results}

Reliability of Judgments. Table 4 presents the indices of reliability and consistency for the three sets of stimulus objects. A comparison with Table 1 indicates that despite the alteration of the configurations for two of the sets, the relative magnitudes of consistency and reliability remained about the same. As in Ex- 
Table 5. Proportion of the variance of scalar products accounted for by the first two Euclidean vectors. Experiment $\mathrm{II}$.

\begin{tabular}{lccc} 
& \multicolumn{3}{c}{ Stimulus Set } \\
& I. Colors & II. Attneave & III. Shopard \\
\hline Modian: & $96.2 \%$ & $95.8 \%$ & $89.0 \%$ \\
Worst: & $92.0 \%$ & $64.4 \%$ & $63.0 \%$ \\
Best: & $99.6 \%$ & $99.0 \%$ & $99.9 \%$ \\
\hline
\end{tabular}

periment I, the similarity judgments for colors appeared to be somewhat less consistent than the judgments for two types of geometric stimuli ( $p$ was .00013 by the Friedman rank test on differences in relative error). But in absolute magnitude, the overall reliability seems to be equivalent for all three sets. At any rate the overlap on these indices was such as to rule out differences in reliability as a basis for understanding other differences between the sets.

Goodness of Fit. Table 5 presents for Experiment II the same information on relative goodness of fit to the Euclidean model as does Table 2 for Experiment $I$. Again it seems that both the parallelograms and the colors tend to show closer agreement to the Euclidean model by this criterion than does the Shepard set. This time, these differences cannot be attributed to
Table 6 Deviations from Euclidean distance: Average deviation of bidimensional minus average deviation of unidimensional judgments. Experiment II.

\begin{tabular}{lccc} 
& I. Colors & II. Attneave & III. Shepard \\
\hline $\begin{array}{l}\text { Mean difference } \\
\begin{array}{l}\text { Pooled critical } \\
\text { ratio (Mann-Whitney) }\end{array}\end{array}$ & $1.4 \mathrm{~cm}$ & $9.0 \mathrm{~cm}$ & $9.6 \mathrm{~cm}$ \\
\hline
\end{tabular}

** Significant at less than 01 level.

differences in configuration. The differences were not statistically significant, even though for five out of six Ss the worst fit was with the Shepard stimuli.

Systematic Deviations from the Euclidean Model. Table 6 presents for Experiment II the data which are comparable to those for Experiment $I$ in Table 3. Since the configurations of the stimulus sets were equivalent in Experiment II, the relative magnitude of these differences between bidimensional and unidimensional comparisons were no longer confounded with differences in number and configuration of objects. The outcome on this criterion was approximately the same as for Experiment I for both colors and Shepard circles. But with the changed configuration, the Attneave parallelograms in Experiment II deviated much more from the Euclidean metric in the direction of the city block (a)

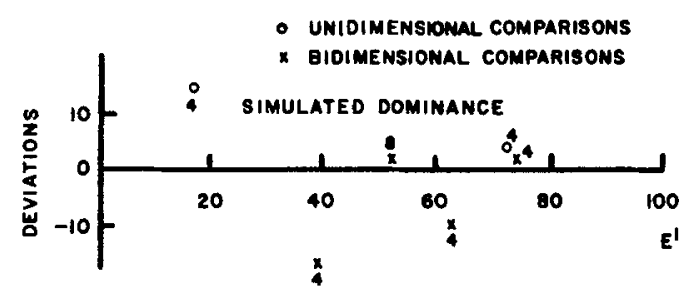

(b)

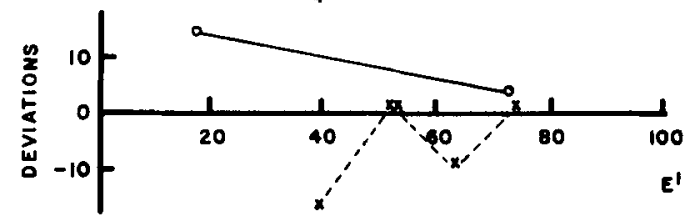

(c)

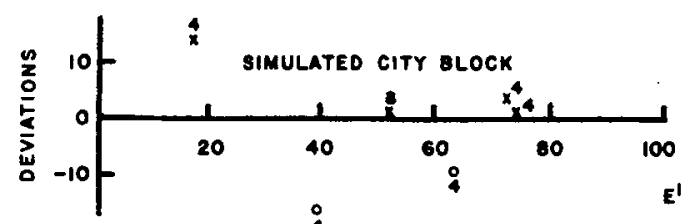

(d)

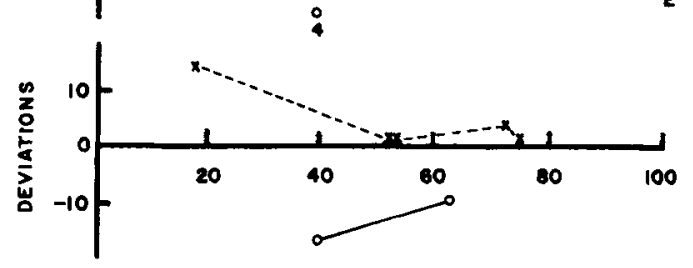

(e)

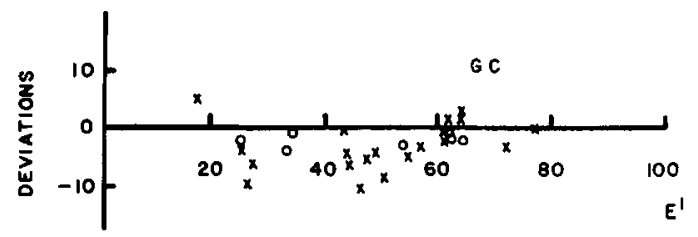

(f)

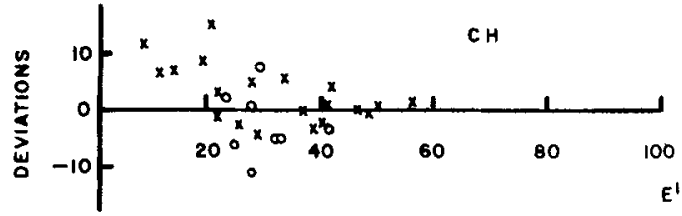

(g)

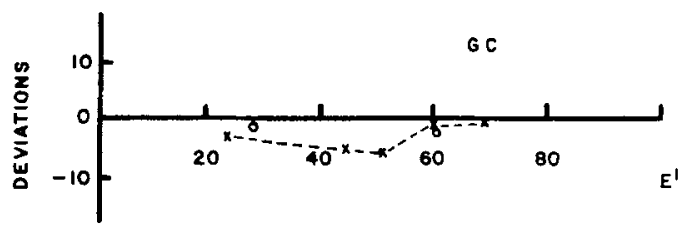

(h)

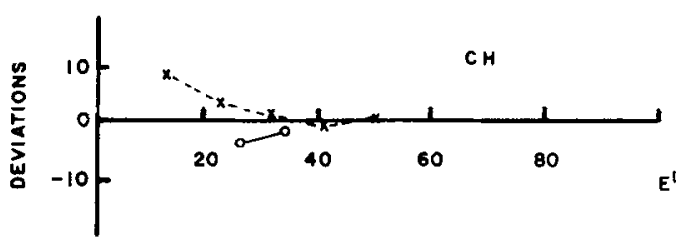

Fig. 7. Pattem of deviations from the Euclidean model for simulated and actual Ss on the color patches (Experiment II): (a) deviations of judgments from the recovered distances for a simulation of the duminance model; (b) the trend of these deviations for the unidimensional and bidimensional judgments fitted by the method of averages: (c) deviations of judgments from the recovered distances for a simulation of the city block model; (d) the trend of these deviations (or the unidimensional and bidimensional judgments fitted by the method of averages; (e) and (f) the actual deviations of two representative $S s$; $(g)$ and $(h)$ the trend of the unidimensional and bidimensional deviations for these two Ss fitted by the method of averages. 


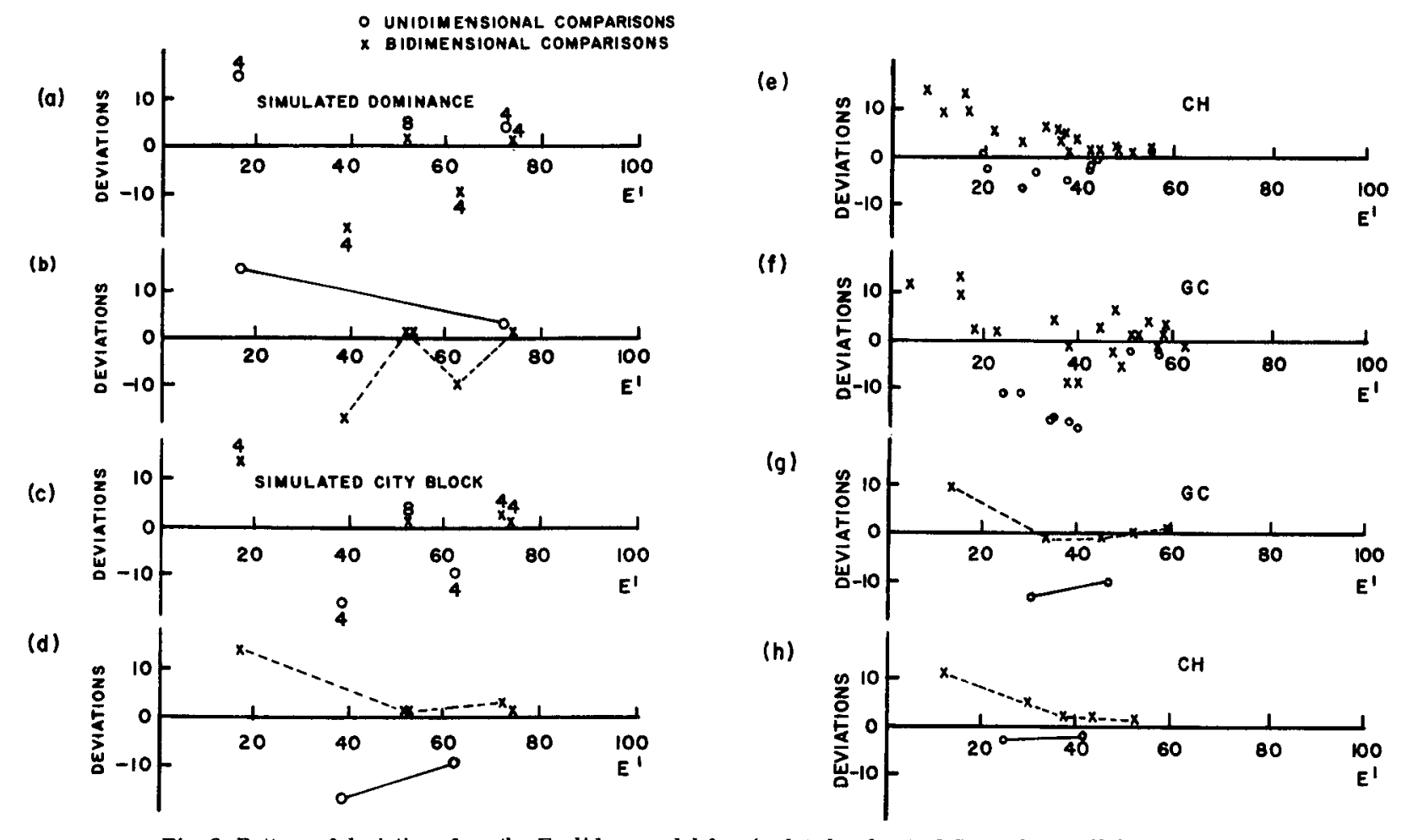

Fig. 8. Pattem of deviations from the Euclidean model for simulated and actual Ss on the parallelograms (Experiment II): (a) deviations of judgments from the recovered distances for a simulation of the dominance model; (b) the trend of these deviations for the unidimensional and bidimensional judgments fitted by the method of averages; (c) deviations of judgments from the recovered distances for a simulation of the city block model; (d) the trend of these deviations for the unidimensional and bidimensional judgments fitted by the method of averages; (e) and ( $f$ ) the the actual deviations of the judgments for two $\mathrm{Ss}$; $(\mathrm{g})$ and $(\mathrm{h})$ the trend of the unidimensional and bidimensional deviations for these two ss fitted by the method of averages.

model. The overall results for the colors again were consistent with the Euclidean model, although two individual Ss seem to have results which were more consistent with the city block model. The critical ratio for one $S$ was significant at the .05 level and just missed the .05 level for the other. However, three of the six Ss had critical ratios which deviated nonsignificantly in the direction towards the dominance model and opposite to the city block.

On the Attneave parallelograms four of the six Ss had positive deviations which were individually significant at .01 level. The other two Ss had deviations very close to zero (.07 and -0.4$)$ and consequently, on this criterion, appeared quite compatible with the Euclidean metric. Three of the six Ss had positive deviations on the Shepard stimull which were individually significant at .01 level, although all of the Se had positive deviations (towards the city block metric). Thus, these results fully corroborate the findings on this criterion from Experiment I. The effect of making the stimulus configurations comparable seems to have been to sharpen the differences in the appropriate spatial models for the colors and the geometric forms.

Pattern of Deviations from the Euclidean Model: Qualitative. Figures 7,8 , and 9 illustrate the same kind of qualitative comparisons between simulated and actual Ss as was made for Experiment $I$ in Figs. 3, 4, and 5. In Fig. 7, for example, the judgments of GC deviated from the Euclidean model somewhat towards the pattern expected of the dominance model (Fig. 7b). On the other hand, the data of $\mathrm{CH}$ deviated towards the city block model (Fig. 7d). The graphs for these same two Ss in Fig. 8 looked just like all but two of the Ss on the Attneave stimuli. The two deviant Ss had graphs consistent with the Euclidean model. The graphs in Fig. 9 were typtcal of Ss whose data we consider in agreement with the city block model (this includes all but one of the six Ss). The negative slopes for $\mathrm{CH}$ reveal that this $S$ tended to exaggerate the distances that were close to the standard relative to the distances that were far from the standard. The vertical separation in the lines fitted to the unidimensional and bidimensional deviations, on the other hand, represents the effects of discrepancy from the spatial model.

By inspection of such graphs, independent of knowledge of the other criteria, we classified, in the color condition, two Ss as agreeing with the dominance model, two as agreeing with the Euclidean model, and two as agreeing with the city block pattern. Using 
the same procedure with the Attneave stimuli, we classified two Ss as Euclidean and four as city block. One $S$ was classified as Euclidean in the Shepard condition and the other five were classified as city block.

Direct Classification of Trials. As we have already mentioned, the configuration of the stimulus sets in Experiment II allowed us to make a separate assessment of S's combinatorial rule for each of the eight trials within a stimulus set. The procedure is the one implied in Fig. 1. We devised a classification rule based on three reference points computed from the two comparison stimuli which differed from the standard on only one of the dimensions. We can use the example in Fig. 1 to illustrate the procedure. The setting of $\mathrm{C1}$ is $30 \mathrm{~cm}$; while the setting of the other unidimensional comparison, $\mathrm{C2}$, is $40 \mathrm{~cm}$ from the standard. If the stimulus corresponding to C3 for this trial had been set between 40 (the value predicted by the dominance model) and $\mathbf{5 0}$ (the value predicted by the Euclidean model) the setting for this trial would be classified as $D$ (dominance) or $E$ (Euclidean) depending upon whether the actual setting was closer to 40 or 50 . If $\mathrm{C3}$ had been set somewhere between 50 and 70 (the value predicted from the city block model), the setting would be classified as $\mathrm{E}$ or $\mathrm{CB}$ (city block) depending upon whether the setting was closer to 50 or 70 . A setting was classified as DV (violation of the Minkowski metric at the dominance end of scale) if the setting was below the predicted D setting by an amount more than half the distance between the predicted $E$ and $D$ settings (in this example, the setting would be classified as DV if it were below 35 ). Similarly, a setting would be classified as TV (violation of triangle inequality) if it were above the predicted $\mathrm{CB}$ setting by more than half the distance between $E$ and $C B$ settings (in this case a setting beyond 80 would be classified as TV).

Using this criterion, we classified S's judgments for each stimulus set by finding the median classification of his eight trial-settings on the ordinal scale DV, D, E, CB, TV. Table 7 presents the results of the application of this criterion. These results support the conclusions reached by our other criteria. Within each stimulus set, moreover, the ranking on the ordinal scale of models and the magnitude of the deviation criterion (Table 6) agreed with one another. The rank order correlations are $.83,1.00$, and .77 within the color, Attneave, and Shepard stimulus sets, respectively. Beyond agreeing with the relative ordering of the stimulus sets in terms of direction of deviation from the (a)

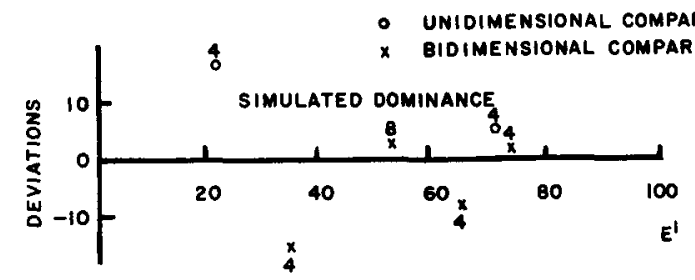

(b)

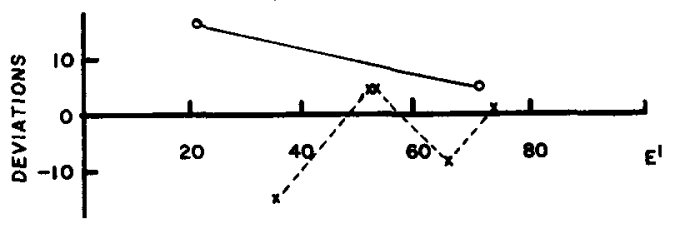

(c)

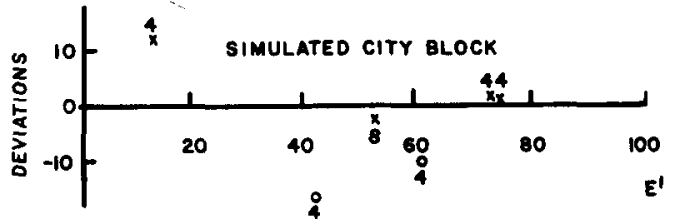

(d)

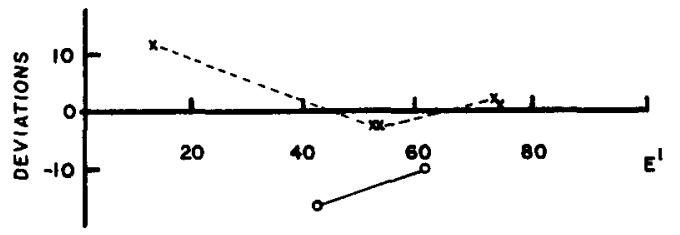

(e)

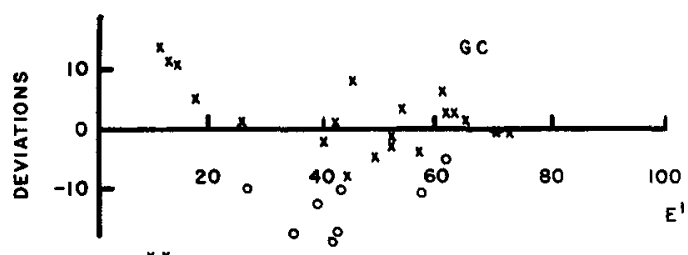

(f)

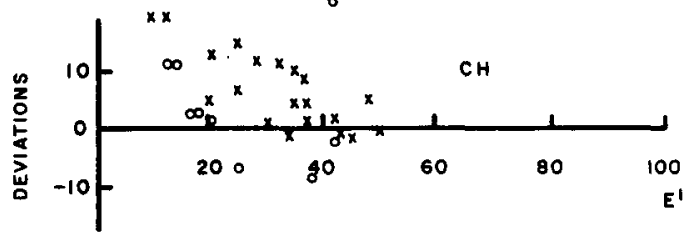

(g)

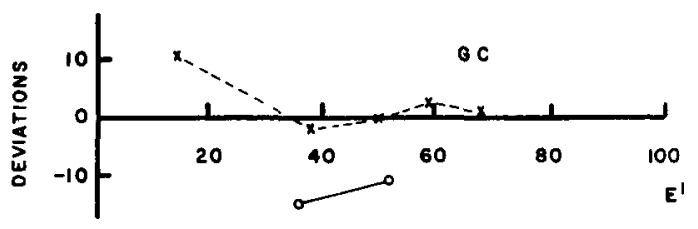

(h)

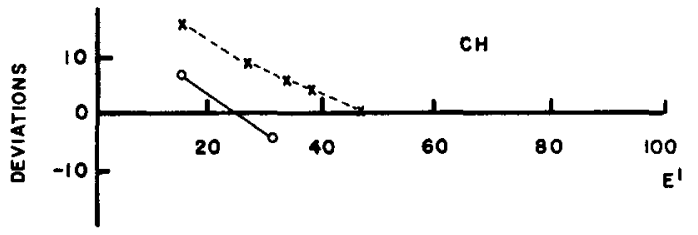

Fig. 9. Pattern of deviations from the Euclidean model for simulated and actual Ss on the Shepard circles-withradius (Experiment II): (a) deviations of judgments from the recovered distances for a simulation of the dominance model; (b) the trend of these deviations for the unidimensional and bidimensional judgments fitted by the method of averages; (c) deviations of judgments from the recovered distances for a simulation of the city block model; (d) the trend of these deviations for the unidimensional and bidimensional judgments fitted by the method of averages; (e) and (f) the actual deviations of the judgments of two Ss; (g) and (h) the trend of the unidimensional and bidimensional deviations for these two Ss fitted by the method of averages. 
Table 7. The relevant spatial model for each stimulus set and each $S$ as determined by a direct analysis of $S^{\prime} s$ settings on each trial. $a$ Experiment II.

\begin{tabular}{lccc} 
Subject & I. Colors & $\begin{array}{c}\text { Stimulus Set } \\
\text { II. Attneave }\end{array}$ & III. Shepard \\
\hline TB & E & TV & TV- \\
GW & D & E & CB- \\
GC & E- & CB & CB \\
RF & CB & DE & E \\
CH & ECB & E & CB \\
WR & D & CB- & CB \\
Median: & E & E plus & CB \\
\hline
\end{tabular}

a Cade: The classifications are considered to be on an ordered scale of models (cf.,Fig. 1) as follows: DV (violation of Minkowski metric), $D$ (dominance), $E$ (Euclidean), $C B$ (city block), TV (violation of Minkowski metric and triangle inequality. A minus or plus sign indicates that the median is slightly above or below the given point on this ordinal scale: a combination of models (such as ECB) indicates that the median is right on the borderline between these two models.

Euclidean model, this latter criterion supplies us with information about the approximate value of the exponent in the Minkowski equation. The colors do, in fact, tend to center around the Euclidean metric, although the indication is that for any individual $S$, the spatial model can be anywhere over the entire range-from dominance to city block. In fact, only two out of six Ss actually were classified by this criterion as being Euclidean in their color judgments. The central tendency for the Attneave stimuli suggests a metric somewhere in between the Euclidean and the city block. Here, again, we have a wide range of models for individuals, going from Euclidean to a violation of the triangle inequality. Outside of the data for one $S$, the Shepard circles yielded similarity judgments that closely fit the city block model. Even in the case of the one apparent exception, his data deviated towards the city block model, and he so strongly emphasized one dimension that there is some question as to whether it would be possible to detect a non-Euclidean pattern in his data even if his judgments had fit this model.

\section{Conclusions to Experiment II}

The results of Experiment II corroborate and amplify the results of Experiment I. In addition, they indicate that the differences in appropriate spatial models between the color patches and geometric forms cannot be attributed to differences in number or configuration of the stimulus sets. Indeed, the equating of the stimulus sets for number and configuration seems to have sharpened the differences, especially in the case of the Attneave parallelograms.

The addition of classifications based on direct application of the Pythagorean theorem to individual trials enables us to pinpoint the approximate value of the exponent in the Minkowski r-space. In Experiment I, for example, our major criterion restricted us to one of three decisions: (1) the set of judgments are consistent with the Euclidean model; (2) the set differs from the Euclidean model in the the direction of a greater exponent (towards the dominance model); (3) the set differs from the Euclidean model in the direction of a smaller exponent (towards the city block). The addition of the new criterion enabled us to indicate more than just the direction in which a set of judgments deviates from the Euclidean. In the case of the Attneave parallelograms, for example, the central tendency over the six Ss was consistent with a metric somewhere between the Euclidean and the city block. For the Shepard circles, the appropriate metric centered squarely on the city block.

Gratifyingly, the various criteria-the average deviations of bidimensional and unidimensional judgments, the qualitative comparison of simulated with actual deviations, and other direct classification of trials -agreed quite closely with one another.

\section{DISCUSSION}

Our findings are consistent with the conclusions drawn from the preceding experiments of Attneave (1950) and Torgerson (1952). By including the different types of stimulus material within one experiment, we have eliminated some possible artifacts due to differences in populations, judgment tasks, and criteria for deciding which is the appropriate spatial model. In particular, it now seems that artifacts due to pooling over Ss (cf., Shepard, 1964), use of psychophysical or scaling procedure, and differences in the configuration and number of stimulus objects can be ruled out. Our results further suggest that the fluctuations of "attention" that characterized Shepard's results are not a factor in the present experiment. Fluctuations or wide shifts in using the component dimensions were virtually precluded because the method employed in the present experiments involved displaying the entire set of stimulus objects on every trial.

The suggestions by Torgerson(1958), Attneave (1962), and Shepard (1964) that the difference in spatial models results from an intrinsic property of the stimulus materials now seems even more plausible. In particular, these authors point towards the phenomenological "obviousness" or "perceptual distinctness" of the component dimensions for the geometric forms as opposed to the perceptual homogeneity and unanalyzability of the component dimensions for the color patches. Presumably, empirical tests could be devised to see if the relevant condition is the perceptual separability of the component dimensions or some other property of the perceptual material such as the qualitative nature of the dimensions or attributes. 3

Closely related to the perceptual separability of the component dimensions is the question of the relative independence of the dimensions. As a check 
on the degree of independence of the component dimensions, we devised a crude criterion. The configuration of each stimulus set in Experiment II on the two dimensions was that of a regular octagon (see Fig. 6). If $\mathrm{S}$ is using the dimensions independently, the judged distance between the objects making up the parallel sides of the octagon should be the same. The distance between the two objects at the lowest saturation, for example, should be the same as the distance between the two objects at the highest saturation, since in both cases the pair of objects are separated by identical amounts on the brightness scale. We computed the actual absolute discrepancy between these two judgments as one part of our crude measure. To this we added the absolute discrepancy between the two pairs that differed by the same amount on saturation but were at different levels of brightness.

With one or two exceptions, all in the color judgments, these discrepancies were quite small, indicating that Ss were using the separate dimensions relatively independently. However, this index of interaction between the dimensions was consistently higher for all Ss with the color patches than with the geometric objects $(p<.03$ by Friedman rank test). Moreover, a $t$ test against the hypothesis that the deviations (algebraic) between dimensions was zero reached statistical significance only for the color patches $(\mathrm{p}<.01)$.

The color patches, then, differ from the geometric figures both in the spatial model that describes the similarity judgments they yield and in the degree of interaction between their component dimensions. Are these two factors more than accidentally related? Some evidence might come from looking at how variations in interaction go along with variations in the spatial model within each stimulus set. Within each stimulus set, then, we rank ordered the six Ss in terms of the closeness of their classification to the dominance model and in terms of the amount of interaction their judgments exhibited on the component dimensions. The rank order correlations were .89 , .82, and .90 within the color, Attneave, and Shepard sets, respectively. Although only six cases were involved, and although the range of variation in interaction was very small in the geometric sets, the two higher correlations are significant at the .05 level (two-tailed) and the lowest correlation has a probability of approximately .06 (two-tailed). Thus, the data strongly suggest that the more the two component dimensions interact, the more the appropriate spatial model will deviate from the city block towards the dominance end of the Minkowski continuum.

If perceptual distinctness of dimensions is the relevant determinant, then we might find it fruitful to look upon the differences in judging distances of color patches and of geometric forms as a problem in information extraction. As Fig. 1 suggests, as the value of $\mathbf{r}$ in the Minkowski equation varies from infinity through 1 , the amount of information that $S$ employs from the two component dimensions increases from a minimum based on only one component to a maximum based on the sum of both components. This observation, when combined with the fact that S's judgments come closest to the city block model when he deals with the components independently, raises questions about the relation of spatial models to informational processing. Does the apparent fit to the Euclidean metric in many judgment situations, for example, indicate that $S$ is having trouble in extracting the information from both dimensions? Do the dimensions tend to mask each other in the color patches? Would speeding up the judgment process or otherwise overloading $S$ result in judgments of the geometric forms that also fit the Euclidean metric?

Perhaps a more basic question is, how should we construe this demonstrated difference in the patterns of judgments to the color patches and the geometric forms? Should we construe it to mean that we are dealing with two qualitatively different judgment situations? The two spatial models, for example, are qualitatively different in that only the Euclidean space possesses the property of rotational invariance. If the axes are rotated in a city block space, the distances between pairs of points change.

Or should we assume that only one of these models is basic and that the other one is either an artifact or is appropriate only in anomalous situations? Torgerson (1958) and Hake and Rodwan (1966) both suggest that the Euclidean model is the more fundamental and basic one. Such an argument is made partly on such grounds that in everyday judgments the component dimensions are rarely obvious and that the property of rotational invariance which is peculiar to the Euclidean space is a highly adaptive property for an organism that seeks invariance and stability in its perceptual world. Another reason for preferring the Euclidean model is that appropriate algorithms and mathematical procedures are readily available only for statistical and geometric models that assume a Euclidean space.

On the other hand, arguments can be made for considering the city block model as being the more fundamental of the two. Additivity, in many areas of science, is acknowledged as a desirable property of models and measurement situations. Householder and Landahl (1945) have provided theoretical reasons for expecting a city block model to be appropriate. In the data from Experiments I and II, a careful examination of the trial-by-trial judgments for the color patches suggests that they are bimodal in comparison with the judgments for the geometric stimuli. In particular, one can get the impression that the similarity judgments for the color patches result from a mixture of two different combinatorial rules. On most comparisons, it appears that $S$ may be making 
his judgment on the basis of just one of the component dimensions. This would correspond to the dominance model in which one dimension dominates or suppresses the other. In a minority of judgments, ones in which $S$ seems to use both dimensions, $S$ appears to follow the city block model. Presumably a mixture of these two types of judgments in the same set of data might lead to an overall pattern that is best described by a compromise model-namely, the Euclidean. At this point, this suggestion must be considered as speculative, since our data do not allow an unequivocal answer on the issue.

Still another possibility is that no one of these spaces has a unique status. Instead, we may have a range of spaces, any one of which could characterize a particular S's judgments with a given set of stimuli at a given point in time. Shepard (1964), for example, interprets his data as pointing to a space in which $r$ is neither 2 nor 1 , but some value in between. And the variation in patterns for our individual Ss could be interpreted as legitimate variations in the true value of $r$, rather than as random deviations from one of the two special values. As was suggested earlier, the value of $r$ that was found to be appropriate in a particular situation might also indicate the ability of $S$ to use the information from the component dimensions in that situation.

Given the possibility that we are dealing with a continuum of combinatorial rules that vary in efficlency of informational usage, the question must be raised: Why should this continuum be conceived in terms of a family of spatial models? And the answer, it seems, is that there is no compelling reason at this time to treat the similarity judgments in terms of a spatial model. Granted, it would be convenient, for example, if a spatial model such as the Euclidean were appropriate. The Euclidean model has served us well, not as a description of S's judgments, but rather as a baseline against which to compare patterns of deviations. But spatial models carry with them a host of assumptions and connotations. Most of these features of the models as yet have no counterparts or demonstrated correspondences in similarity judgments. Almost surely much simpler models or combinatorial rules can be found which will describe the judgments just as well, but without the heavy excess of extra assumptions and implications. For example, a simple linear equation which can take on two states -one for bidimensional and one for unidimensional judgments-could probably describe the data very well. At this stage of the research on similarity judg- ments, it would be premature to take a stand relative to the appropriateness of a spatial or a nonspatial model. Since Attneave's classic study in 1950, surprisingly little in the way of empirical knowledge has been collected on similarity judgments. The mathematical models and their accompanying machinery far outrun any hard data on how S actually makes his judgments. What is needed at this time is more and better empirical data on how $S$ does go about making his judgments.

\section{References}

Attneave, F. Dimensions of similarity. Amer. J. Psychol., 1950, $63,516-556$.

Attneave, F. Perception and related areas. In S. Koch (Ed.), Psychology: a study of a science, Vol. 4. New York: McGraw-Hill, 1962, 619-659.

Hake, H. W., \& Rodwan, A. S. Perception and recognition. In J. B. Sidowski (Ed.), Experimental methods and instrumentation in. psychology. New York: McGraw-Hill, 1966, 331-381.

Helm, C. E. Multidimensional ratio scaling of perceived color relations. J. Opt. Soc. Amer., 1964, 54, 256-262.

Helm, C. E., \& Tucker, C. R. Individual differences in the structure of color perception. Amer. J. Psychol., 1962, 75, 437-444.

Householder, A. S., \& Landahl, H. D. Mathematical biophysics of the central nervous system. Bloomington, Indiana: Principia Press, 1945.

Indow, $\mathbf{T}$. Two kinds of multidimensional scaling methods as tools for investigating color space from the macroscopic point of view. Acta Chromatica, 1963, 1, 60-71.

Indow, $\mathbf{T}$, \& Kanazawa, $\mathbf{K}$. Multidimensional mapping of Munsell colors varying in hue, chroma, and value. $J$. exp. Psychol., $1960,59,330-336$.

Indow, T., \& Uchizono, T. Multidimensional mapping of Munsell colors varying in hue and chroma. J. exp. Psychol., 1960, 59, 321-329.

Mosteller, F., \& Bush, R. R. Selected quantitative techniques. In G. Lindzey (Ed.), Handbook of social psychology, Vol. 1. Cambridge, Mass.: Addison-Wesley, 1954. 289-334.

Richardson, M. W. Multidimensional psychophysics. Psychol. Bull., 1938, 35, 659-660. (Abstract)

Shepard, R. N. Attention and the metric structure of the stimulus space. J. math. Psychol., 1964, 1, 54-87.

Torgerson, W. S. Multidimensional scaling: I. Theory and method. Psychometrika, 1952, 17, 401-419.

Torgerson, W. S. Theory and methods of scaling. New York: Wiley, 1958.

\section{Notes}

1. This research was supported by Public Health Research Grant MH 11644 from the National Institute of Health.

2. This was the fourth tray from the top for the nine color chips and the eight circles; it was the third tray from the top for the seven parallelograms.

3. We have completed such a study and will report on it in a future paper.

(Accepted for publication March 7, 1967.) 